\title{
Comparative Transcriptome Analysis of Queen, Worker, and Larva of Asian Honeybee, Apis cerana
}

\author{
Woo Jin Kim${ }^{1}$, Seok Hee Lee ${ }^{1}$, Saes Byeol An ${ }^{1}$, Song Eun Kim ${ }^{1}$ Qin $\mathrm{Liu}^{1}$, Jae Young Choi ${ }^{2}$, \\ and Yeon $\mathrm{Ho} \mathrm{Je}^{12 *}$ \\ ${ }^{I}$ Department of Agricultural Biotechnology, College of Agriculture and Life Science, Seoul National University, Seoul, Korea \\ ${ }^{2}$ Research Institute for Agriculture and Life Sciences, Seoul National University, Seoul, Korea
}

\section{Abstract}

The Asian honeybee, Apis cerana, is a native honeybee species in Korea which is important in agriculture for pollination and honey production. For better understanding of the physiology of $A$. cerana, high-throughput Illumina transcriptome sequencing was performed to analyze the gene expression profiles of queen, worker, and larva. A total of 219,799,682 clean reads corresponding to $22.2 \mathrm{~Gb}$ of nucleotide sequences was obtained from the whole body total RNA samples. The Apis mellifera reference mRNA sequence database was used to measure the gene expression level with Bowtie2 and eXpress software, and the lllumina short reads were then mapped to 11,459 out of 11,736 A. mellifera reference genes. Total of 9,221 genes with FPKM value greater than 5 of each sample group were subjected to eggNOG with BLASTX for gene ontology analysis. The differential gene expression between queen and worker, and worker and larva were analyzed to screen the overexpressed genes in each sample group. In the queen and worker sample group, total of 1,766 genes were differentially expressed with 887 and 879 genes overexpressed over two folds in queen and worker, respectively. In the worker and larva sample group, total of 1,410 genes were differentially expressed with 1,009 and 401 genes overexpressed over two folds in worker and larva, respectively.

(C) 2013 The Korean Society of Sericultural Sciences

Int. J. Indust. Entomol. 27(2), 271-276 (2013)
Received : 5 Dec 2013

Accepted : 20 Dec 2013

Keywords:

Apis cerana,

Transcriptome,

Illumina,

Developmental stage,

Caste

\section{Introduction}

Apis cerana, the Asian honeybee is a native bee species to eastern and southeastern Asian countries such as Korea, China, and Japan. In Korea, A. cerana is one of the most important honeybee species along with western honeybee, Apis mellifera because not only of the economic importance of honey production, but also the importance as one of the pollinator species in agriculture. Also A. cerana had been concerned of its strong resistance to the ectoparasitic mites (Peng et al., 1987). However, the recent outbreak of sacbrood virus (SBV) belonging to the genus Iflavirus which infects $A$. cerana (Choi et al., 2010) caused a devastating colony loss of Korean honeybee industry.

SBV infected larva fails to pupate, and accumulates virus enriched ecdysal fluid beneath its unshed skin (Bailey et al., 1964). SBV also infects adult bees, however, the mortality

\section{${ }^{*}$ Corresponding author.}

Yeon Ho Je

Department of Agricultural Biotechnology, College of Agriculture and Life Science, Seoul National University, Seoul, 151-921 Korea Tel: +82-2-880-4706 / FAX: +82-2-873-2319

E-mail: btrus@snu.ac.kr 
of SBV infected adults is low while SBV results significant larval mortality (Bailey and Fernando, 1972) which suggests that the responses of the hosts against SBV might be different to each other according to their developmental stage and/ or caste. Recently, the SBV isolated from A. cerana in Korea was sequenced, and characterized by molecular genomics and phylogenetic approach to reveal that the SBV found in Korea is different from other strains previously published (Choe et al., 2012a, 2012b), however, the molecular biology and nucleotide sequence information of $A$. cerana, which is very important to study the virus and host interaction, is still limited. Currently, $A$.cerana genome sequence is not available yet, and there are just 115 ESTs and 7,009 amino acid sequences of $A$. cerana are available in the NCBI database which are only $0.2 \%$ and $17 \%$ of A. mellifera based on the number of sequence entries. For comprehensive understanding of the virus and host interaction, accumulation of nucleotide sequence data, and study of functional genomics of $A$. cerana is indispensable.

Illumina sequencing is a recently developed technology which can produce hundreds of millions of short reads from DNA or cDNA sample (Bentley et al., 2008). The high sequencing capacity of Illumina sequencing makes possible tagging of the gene or genome sequences by aligning the shorts reads of transcriptome to reference sequences to measure the gene expression level by counting the number of tags produced from each gene (Mardis, 2008; Velculescu et al., 1995). In this study, we employed the Illumina sequencing technology to sequence cDNA samples of each developmental stages of $A$. cerana, and mapped the short reads to the closely related species, $A$. mellifera reference genes to search and measure stage and caste specific gene transcripts and their transcription levels for studying the candidate genes responsible to their physiological differences.

\section{Materials and Methods}

Insect

The Apis cerana samples were collected from the beehive of honeybee farm at Cheongwon-gun, Chungcheongbuk-do, Korea. The honeybee samples were collected in mid-March 2013, therefore the workers were virtually all winter nurse bees, no forager. Newly laid eggs were found in the bee hive which indicate that the queen was actively laying eggs. One queen, five of workers and approximately seven days old larvae were sampled per group for RNA isolation, respectively. The compound eyes, wings, and legs of the queen and worker samples were removed beforehand, and then the total RNA samples were extracted from whole body of each sample for sequencing.

\section{cDNA library preparation and Illumina tran- scriptome sequencing}

To construct a cDNA library, the total RNA was isolated using Qiazol lysis reagent (Qiagen, Germany) according to the manufacture's protocol. Five of the total RNA samples from the workers and larvae groups were pooled equal amount as one sample of $10 \mu \mathrm{g}$ for transcriptome sequencing, respectively. The integrity of total RNA was examined by using 2100 Bioanalyzer (Agilent, USA) with RNA 6000 nano chip. The mRNA in total RNA was converted into a library of template molecules suitable for subsequent cluster generation using the reagents provided in the Illumina TruSeq RNA Sample Preparation Kit. The first step in the workflow involves purifying the poly-A containing mRNA molecules using poly-T oligo-attached magnetic beads. Following purification, the mRNA was fragmented into small pieces using divalent cations under elevated temperature. The cleaved RNA fragments were copied into first strand cDNA using reverse transcriptase and random primers. This was followed by second strand cDNA synthesis using DNA polymerase I and RNase $H$. These cDNA fragments then went through an end repair process, the addition of a single 'A' base, and ligation of the adapters. The products were then purified and enriched with PCR to create the final cDNA library. Finally the cDNA library of 250 300 bp insert was subjected to Illumina HiSeq 2000 sequencer to sequence the 101 bases of 5' end.

\section{Analysis of transcriptome sequencing results}

The raw Illumina sequence reads were filtered to remove low quality sequences by using NGS QC Toolkit v2.3 (Patel and Jain, 2012) before bioinformatical analysis. The sequence reads of base called with error rate higher than $0.1 \%$ (quality score $<30$ ) were eliminated, and the remaining clean reads were used for further analysis. The filtered Illumina short reads were then mapped to A. mellifera reference mRNA sequences (ftp://ftp.ncbi.nih.gov/ 
Table 1. Summary of the A. cerana transcriptome sequencing

\begin{tabular}{|cccc|}
\hline & Queen & Worker & Larva \\
\hline Total sequence reads & $82,069,132$ & $79,919,960$ & $80,424,924$ \\
\hline Total clean reads & $74,905,753$ & $71,548,307$ & $73,345,622$ \\
\hline Total nucleotides of clean reads & $7,565,481,053$ & $7,226,379,007$ & $7,407,907,822$ \\
\hline Q30 percentage & $92.37 \%$ & $91.03 \%$ & $92.20 \%$ \\
\hline GC percentage & $34.67 \%$ & $39.10 \%$ & $37.33 \%$ \\
\hline
\end{tabular}

genomes/Apis_mellifera/RNA) from the honey bee assembly v4.5 of BeeBase (Munoz-Torres et al., 2011) using Bowtie2 software (Langmead and Salzberg, 2012) with default parameters. The mapping results of each sample to reference mRNA sequences obtained by Bowtie 2 were then quantified by eXpress software (Roberts and Pachter, 2013). The gene expression profiles of each sample obtained by eXpress were calculated as fragments per kilobase of exon per million reads (FPKM) value to compare the gene expression levels between samples.

\section{Gene ontology analysis}

The reference mRNA sequences which were mapped with the Illumina short reads by Bowtie 2 software were subjected to eggNOG (evolutionary genealogy of genes: Non-supervised Orthologous Groups) (Jensen et al., 2008) by BLASTX with a cut-off E-value of $10^{-5}$ for gene ontology (GO) anlaysis. The returned mRNA sequences above cut-off score were annotated and categorized for further analysis.

\section{Results and Discussion}

\section{Transcriptome sequencing}

The Illumina sequencing generated a total of 242,414,016 single-end raw reads, and a total of 219,799,682 reads which was $90.67 \%$ of the raw reads was obtained after Q30 (sequencing error rate, $0.1 \%$ ) filtration for further analysis. The accumulated length of the total filtered reads was 22,199,767,882 bases with GC percentage of $37.0 \%$. The detailed transcriptome sequencing results of each sample group are summarized in Table 1. The raw Illumina sequencing results were submitted to NABIC (National Agrucultural Biotechnology Information Center, Rural
Table 2. Detailed statistics of the gene expression levels of each sample

\begin{tabular}{|cccc|}
\hline & Queen & Worker & Larva \\
\hline Max FPKM & $328,145.853$ & $324,600.660$ & $1,393,940.143$ \\
\hline Min FPKM & 0.0095 & 0.0098 & 0.0023 \\
\hline Avg FPKM & 226.5836 & 248.9488 & 339.8804 \\
\hline Median FPKM & 21.6844 & 20.1978 & 14.8157 \\
\hline $\begin{array}{c}\text { No. of mapped } \\
\text { genes }\end{array}$ & 10,197 & 10,129 & 10,090 \\
\hline
\end{tabular}

Development Administration, Korea) NGS SRA database. The accession numbers for the transcriptome of Queen, workers, and larvae are NN0646, NN0650, and NN0648, respectively.

\section{Analysis of gene expression level}

The Illumina short reads of each A. cerana sample were mapped to $A$. mellifera reference mRNA sequences by Bowtie2 software. Total of 11,459 out of 11,736 A. mellifera reference sequences were mapped with the short reads of $A$. cerana transcriptome, and the expression levels of each gene were converted to FPKM value by eXpress software. Detailed statistics of the gene expression levels of each sample group are summarized in Table 2. The larva sample includes genes with remarkably higher FPKM value than those of queen and worker samples, and those genes were ribosomal protein genes which are part of the essential cellular machinery for protein synthesis, development, and growth.

\section{Gene ontology analysis of differentially expressed genes}

To compare the gene expression profiles, the FPKM values 
of each gene were then arithmetically normalized based on the ratio of the total numbers of Q30-filtered short reads between sample groups. The genes which were overexpressed over twofold between the two sample groups were isolated, and the sum of FPKM value of each gene was calculated. A total of 9,221 genes with sum of FPKM value greater than 5 were subjected to eggNOG analysis. The eggNOG functional groups and their abbreviations are as follows; [Q] Secondary metabolites biosynthesis, transport and catabolism, [P] Inorganic ion transport and metabolism, [I] Lipid transport and metabolism, [H]

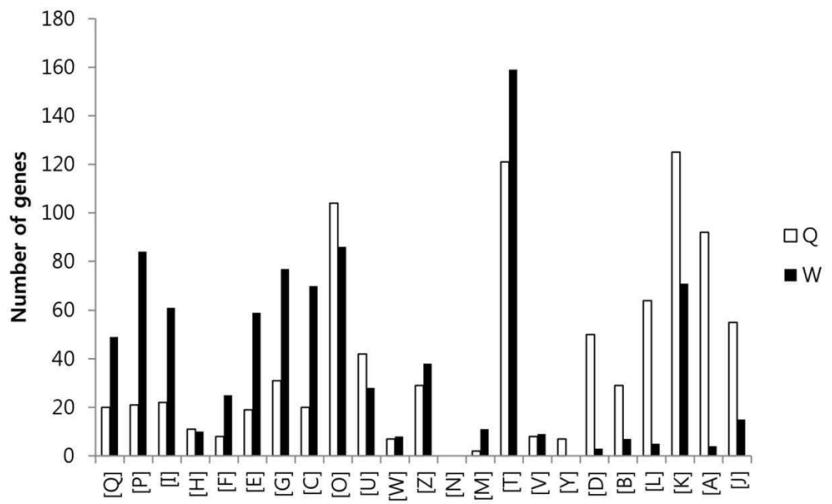

Fig. 1. Graph of eggNOG analysis of the genes which were overexpressed over two-fold in queen and worker. The number of overexpressed genes in the queen and worker of each GO group are indicated as white and black, respectively.
Coenzyme transport and metabolism, [F] Nucleotide transport and metabolism, [E] Amino acid transport and metabolism, [G] Carbohydrate transport and metabolism, [C] Energy production and conversion, [O] Posttranslational modification, protein turnover, chaperones, [U] Intracellular trafficking, secretion, and vesicular transport, [W] Extracellular structures, [Z] Cytoskeleton, [N] Cell motility, [M] Cell wall/membrane/ envelope biogenesis, [T] Signal transduction mechanisms, [V] Defense mechanisms, [Y] Nuclear structure, [D] Cell cycle

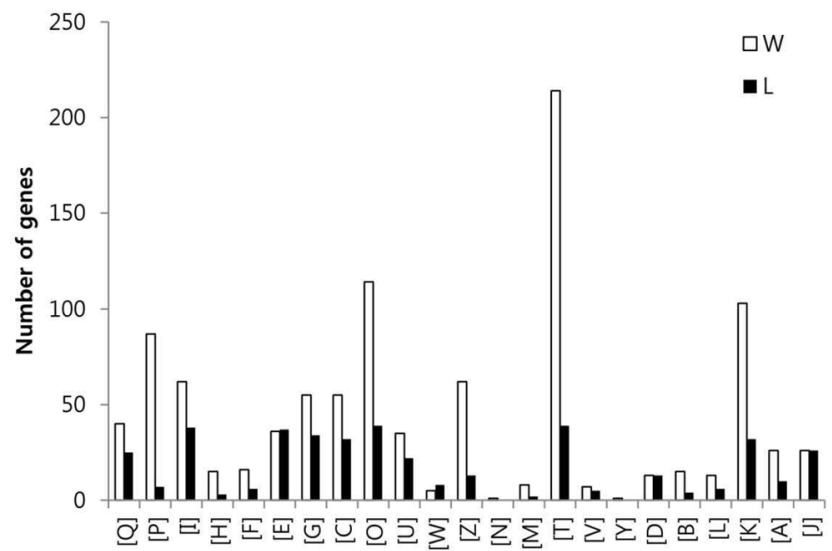

Fig. 2. Graph of eggNOG anlaysis of the genes which were overexpressed over two-fold in worker and larva. The number of overexpressed genes in the worker and larva of each GO group are indicated as white and black, respectively.

Table 3. The differentially express genes over 500 folds in queen and worker.

\begin{tabular}{|ccccccc|}
\hline $\begin{array}{c}\text { A. mellifera } \\
\text { Ref gene ID }\end{array}$ & $\begin{array}{c}\text { UniprotKB } \\
\text { accession no. }\end{array}$ & Gene function & $\begin{array}{c}\text { eggNOG } \\
\text { category }\end{array}$ & $\begin{array}{c}\text { FPKM } \\
\text { (Queen) }\end{array}$ & $\begin{array}{c}\text { FPKM } \\
\text { (Worker) }\end{array}$ & Q/W ratio \\
\hline CL10988Contig1 & XP_394161 & Orthodenticle-1 protein & $\mathrm{K}$ & 183.198 & 0.069 & 2659.336 \\
\hline CL8765Contig1 & XP_394847 & Gooseberry protein & $\mathrm{K}$ & 18.735 & 0.011 & 1766.998 \\
\hline CL52367Contig1 & XP_001119912 & Transcription factor & $\mathrm{K}$ & 66.151 & 0.041 & 1625.326 \\
\hline CL8225Contig1 & XP_001122398 & $\begin{array}{c}\text { Protein involved in genital } \\
\text { disc development }\end{array}$ & $\mathrm{K}$ & 371.386 & 0.425 & 873.237 \\
\hline CL47583Contig1 & XP_001601536 & $\begin{array}{c}\text { Protein involved in regulation } \\
\text { of transcription from }\end{array}$ & $\mathrm{K}$ & 474.849 & 0.681 & 697.110 \\
\hline RL46357Contig1 & XP_624026 & Cytochrome P450 protein & $\mathrm{Q}$ & 85.404 & 0.123 & 692.136 \\
\hline CL52111Contig1 & Q17061 & Major royal jelly protein & $\mathrm{S}$ & 2.074 & 4237.383 & 0.001 \\
\hline CL23420Contig1 & XP_394370 & Serine-Type enodpeptidase & $\mathrm{O}$ & 4.599 & 6503.741 & 0.000 \\
\hline CL48693Contig1 & XP_001121120 & Cysteine-Rich venom protein & $\mathrm{S}$ & 0.520 & 683.745 & 0.001 \\
\hline CL49002Contig1 & XP_001121120 & Cysteine-Rich venom protein & $\mathrm{S}$ & 0.640 & 400.828 & 0.002 \\
\hline CL778Contig2 & Q9U8X6 & Glucose oxidase & $\mathrm{C}$ & 0.194 & 119.962 & 0.002 \\
\hline
\end{tabular}


Table 4. The differentially express genes over 500 folds in worker and larva.

\begin{tabular}{|ccccccc|}
\hline $\begin{array}{c}\text { A. mellifera } \\
\text { Ref gene ID }\end{array}$ & $\begin{array}{c}\text { UniprotKB } \\
\text { accession no. }\end{array}$ & Gene function & $\begin{array}{c}\text { eggNOG } \\
\text { category }\end{array}$ & $\begin{array}{c}\text { FPKM } \\
\text { (Worker) }\end{array}$ & $\begin{array}{c}\text { FPKM } \\
\text { (Larva) }\end{array}$ & W/L ratio \\
\hline CL778Contig2 & Q9U8X6 & Glucose oxidase & C & 119.962 & 0.010 & 11811.665 \\
\hline CL21074Contig1 & O97432 & Major royal jelly protein & S & 11022.810 & 1.227 & 8980.947 \\
\hline CL9379Contig1 & Q25BT8 & Alpha-Glucosidase & G & 3070.006 & 0.616 & 4980.110 \\
\hline CL8792Contig1 & XP_397087 & Serine-Type endopeptidase & O & 555.582 & 0.128 & 4330.956 \\
\hline CL14122Contig1 & Q17053 & Rhodopsin protein & T & 259.021 & 0.209 & 1239.556 \\
\hline CL21265Contig1 & Q17060 & Major royal jelly protein & S & 257.226 & 0.263 & 978.909 \\
\hline CL36969Contig1 & NP_001011651 & Troponin C protein & T & 264.119 & 0.282 & 937.569 \\
\hline CL50642Contig1 & Q1W647 & Odorant binding protein 3 & P & 400.704 & 0.456 & 879.473 \\
\hline CL29065Contig1 & O77061 & Major royal jelly protein & S & 1169.244 & 1.339 & 873.426 \\
\hline CL12073Contig1 & XP_001122057 & Leucine-Rich repeats & S & 403.929 & 0.578 & 699.182 \\
\hline CL42234Contig1 & XP_395084 & Neuropeptide Y receptor & T & 0.191 & 1385.589 & 0.000 \\
\hline CL5768Contig1 & A6YLP8 & Hexamerin protein & S & 1.329 & 2487.960 & 0.001 \\
\hline CL46982Contig1 & XP_001121128 & Structural constituent of & S & 5.523 & 6784.944 & 0.001 \\
\hline CL11903Contig1 & XP_397272 & Cytochrome P450 protein & I & 0.036 & 37.487 & 0.001 \\
\hline CL14883Contig1 & Q6J4Q1 & Hexamerin protein & S & 7.903 & 6855.978 & 0.001 \\
\hline CL38195Contig1 & Q1W641 & Odorant-Binding protein & S & 0.936 & 491.532 & 0.002 \\
\hline
\end{tabular}

control, cell division, chromosome partitioning, [B] Chromatin structure and dynamics, [L] Replication, recombination and repair, [K] Transcription, [A] RNA processing and modification, and $[\mathrm{J}]$ Translation, ribosomal structure and biogenesis.

In the comparison of queen and worker, total of 3,014 and 3,009 eggNOG tagged genes from queen and worker were compared their expression levels, and identified total of 887 (22.7\%) and $879(22.5 \%)$ genes which were overexpressed over two folds, respectively. In the comparison of worker and larva, total of 3,492 and 2,884 eggNOG tagged genes from worker and larva were compared, respectively, and identified total of 1,009 (25.9\%) and $401(10.3 \%)$ genes which were overexpressed over two folds, respectively.

The GO distributions demonstrated that the genes related to nutritional metabolism were overexpressed in the worker while the genes related to cell division and nucleic acid processing were overexpressed in the queen. These results reflect the role of each caste which are honey and royal jelly production, and reproduction of worker and queen, respectively (Fig. 1). On the other hand, the genes related to transcription, translation, and transduction were overexpressed in the larva which reflect the highly active genes of developing larvae (Fig. 2). The genes significantly overexpressed over 500-fold are summarized in Table 3 and 4.

\section{Acknowledgment}

This work was supported by a grant from the Next-Generation BioGreen 21 Program (no.PJ009031), Rural Development Administration, Republic of Korea. Seok Hee Lee, Saes Byeol An, Qin Liu, and Song Eun Kim were supported by the Brain Korea 21 project.

\section{Reference}

Bailey L, Fernando EFW (1972) Effects of sacbrood virus on adult 
honey-bees. Ann. Appl. Biol. 72, 27-35.

Bailey L, Gibbs AJ, Woods RD (1964) Sacbrood virus of the larval honey bee (Apis mellifera linnaeus). Virology 23, 425-429.

Bentley DR, Balasubramanian S, Swerdlow HP, Smith GP, Milton J, Brown CG, Hall KP, Evers DJ, Barnes CL, Bignell HR, et al. (2008) Accurate whole human genome sequencing using reversible terminator chemistry. Nature 456, 53-59.

Choe SE, Nguyen LTK, Noh JH, Kweon CH, Reddy KE, Koh HB, Chang KY, Kang SW (2012a) Analysis of the complete genome sequence of two Korean sacbrood viruses in the Honey bee, Apis mellifera. Virology 432, 155-161.

Choe S-E, Nguyen TT-D, Hyun B-H, Noh J-H, Lee H-S, Lee C-H, Kang S-W (2012b) Genetic and phylogenetic analysis of South Korean sacbrood virus isolates from infected honey bees (Apis cerana). Veterinary Microbiology 157, 32-40.

Choi YS, Lee MY, Hong IP, Kim NS, Kim HK, Lee KG, Lee ML (2010)

Occurrence of sacbrood virus in Korean apiaries from Apis cerana (Hymenoptera: Apidae). Korean Journal of Apiculture 25, 187-191.

Jensen LJ, Julien P, Kuhn M, von Mering C, Muller J, Doerks T,
Bork, P. (2008) eggNOG: automated construction and annotation of orthologous groups of genes. Nucleic Acids Res 36, D250-D254.

Langmead B, Salzberg SL (2012) Fast gapped-read alignment with Bowtie 2. Nat Meth 9, 357-359.

Mardis ER (2008) Next-Generation DNA Sequencing Methods. Annual Review of Genomics and Human Genetics 9, 387-402.

Munoz-Torres MC, Reese JT, Childers CP, Bennett AK, Sundaram JP, Childs KL, Anzola JM, Milshina N, Elsik CG (2011) Hymenoptera Genome Database: integrated community resources for insect species of the order Hymenoptera. Nucl. Acids Res. 39, D658-D662.

Patel RK, Jain M (2012) NGS QC Toolkit: A Toolkit for Quality Control of Next Generation Sequencing Data. PLoS ONE 7, e30619.

Peng Y-S, Fang Y, Xu S, Ge L (1987) The resistance mechanism of the Asian honey bee, Apis cerana Fabr., to an ectoparasitic mite, Varroa jacobsoni Oudemans. Journal of Invertebrate Pathology 49, 54-60.

Roberts A, Pachter L (2013) Streaming fragment assignment for realtime analysis of sequencing experiments. Nat Meth 10, 71-73.

Velculescu V.E, Zhang L, Vogelstein B, Kinzler KW (1995) Serial analysis of gene expression. Science 270, 484-487. 